Original Research Paper

\title{
Hydroxyl Radical-Scavenging Activity-Guided Extraction of Peptides from Oviductus ranae
}

\author{
${ }^{1}$ Jieyang Shen, ${ }^{1}$ Yihui Liu, ${ }^{1}$ Jiahao Xu, ${ }^{1}$ Shenbo Ye, ${ }^{2}$ Xiaoxiao Xu, \\ ${ }^{2}$ Yang Liu, ${ }^{1}$ Lixue Zheng and ${ }^{1}$ Yang Zhang \\ ${ }^{I}$ School of Biology and Food Engineering, Changshu Institute of Technology, Changshu, China \\ ${ }^{2}$ School of Chemistry and Pharmaceutical Engineering, Jilin Institute of Chemical Technology, Jilin, China
}

\author{
Article history \\ Received: 08-07-2021 \\ Revised: 16-09-2021 \\ Accepted: 25-09-2021 \\ Corresponding Authors: \\ Lixue Zheng \\ School of Biology and Food \\ Engineering/Changshu Institute \\ of Technology, Changshu, \\ China \\ Email: zhenglixue@cslg.edu.cn \\ Yang Zhang \\ School of Biology and Food \\ Engineering/Changshu Institute \\ of Technology, Changshu, \\ China \\ Email: zhangyang@cslg.edu.cn
}

\begin{abstract}
Oviductus Ranae (OR) is a traditional Chinese medicinal material that is listed in Chinese Pharmacopeia with proteins the most abundant ingredients. In present work, pepsin/trypsin-based stepwise hydrolysis was applied to extract Antioxidant Peptides from OR (APOR) using hydroxyl radical-scavenging capacity as evaluation index. After single-factor experiment, response surface method coupled with Box-Behnken design were used for optimizing process parameters and achieved the optimal conditions: Pepsin amount of $2.0 \%$, pepsin incubation time of $3.5 \mathrm{~h}$, trypsin amount of $2.0 \%$ and trypsin incubation time of $3.0 \mathrm{~h}$, under these conditions, the hydroxyl radical-scavenging rate of obtained APOR was $75.52 \pm 1.92 \%$, which was extremely similar to the predicated value with relative deviation of $\pm 1.42 \%$. The half inhibitory concentration of APOR against hydroxyl radical was $3.71 \pm 0.71 \mathrm{mg} \cdot \mathrm{ml}^{-1}$, which was lower than that of proteins from OR $\left(4.85 \pm 0.06 \mathrm{mg} \cdot \mathrm{mL}^{-1}\right.$, disclosed in our previously published paper). The higher antioxidant capacity of APOR could be related to the presence of rich antioxidant amino acids and relatively lower molecular weights, which deserve to be explored in the next future.
\end{abstract}

Keywords: Oviductus ranae, Antioxidant Peptides, Pepsin/Trypsin-Based Stepwise Hydrolysis, Response Surface Method

\section{Introduction}

Reactive Oxygen Species (ROS), including a variety of unstable free radicals are inevitable products of oxidative metabolism (Kurniawan et al., 2020). These free radicals are widely involved in the regulation of physiological and biochemical functions. However, excessive accumulation of free radicals will oxidize proteins, lipids and DNA to damage cells and organs irreversibly, leading to the onset and aggravation of many diseases and aging (Martinez-Useros et al., 2017). Among different ROS, hydroxyl radical possesses the highest oxidizability to biomacromolecules and has been verified to be closely associated with many oxidative-stress related pathophysiological processes (Hou et al., 2020).

It is found that exogenous supplement of antioxidants is beneficial for inhibiting and delaying lipid oxidation to a certain extent and significantly reduces the damages of excessive free radicals to the body (Gorji et al., 2019), thus it is necessary to study and explore new antioxidants.

Many protein-based antioxidants are limited by the macromolecular structures that hinder the interaction with free radicals (Xia et al., 2012). It is a feasible and common way to obtain peptides with antioxidant potentials by hydrolyzing maternal proteins by different proteases (Zhao, 2018). Due to relatively lower molecular weights, peptides obtained by digesting proteins are more easily absorbed in the gastrointestinal tract and functional properties are improved, as well as allergenicity is reduced (Beverly et al., 2021). Recently, more and more studies have revealed that antioxidant peptides inhibit oxidation via scavenging free radicals, reducing peroxides, inactivating ROS and so on (Wen et al., 2020; Yan et al., 2020). Studies have also confirmed that antioxidant peptides can potentiate the therapeutic effects of non-peptide antioxidants (De Luca et al., 2016; Schroeder et al., 2005).

Rana dybowskii, also known as Chinese brown frog or Hashima, is a famous economic frog species that has been consumed as delicious dish and tonic for a long history in China. It is naturally distributed in northeastern China and artificial breeding of wild $R$. dybowskii has succeeded and reached intensive cultivation in 1990s (Zhang et al., 2019a). The medicinal value of $R$. dybowskii is chiefly 
contributed by the female body's oviduct and its dried product, called Oviductus ranae (OR) is a traditional Chinese medicinal material listed in Chinese Pharmacopeia (Zhang et al., 2017). Traditional Chinese medicine holds the opinion that OR has a power to nourish Yin and moisturize the lung. Modern pharmaceutical studies confirmed that OR possesses various functions relating to traditional uses, such as immune-enhancing properties, antioxidant and anti-fatigue activities. OR comprise several nutrients and bioactive ingredients with proteins the highest content of over $50 \%$ in most cases (Yu et al., 2020; Zhang et al., 2019a-b). Thus in previous work, the antioxidant and anti-fatigue potentials of Proteins from OR (POR) were explore and noted that the in vivo antioxidant capacity of $\mathrm{POR}$ weighs on the in vitro counterpart, which indicated that the underlying antioxidant activity-contributed constituents of POR would be the digestive products in the gastrointestinal tract (Zhang et al., 2019b). This hypothesis inspired us to study the antioxidant possibility of peptides that are obtained by hydrolyzing POR using endogenous digestive enzymes.

Considering that proteins are the dominant nutrient in $\mathrm{OR}$, in present investigation, in order to facilitate operation and save starting materials, we prepared the antioxidant peptides from POR with OR as material directly. To stimulate the in vivo digestive process of proteins, pepsin/trypsin-based stepwise hydrolysis was applied to achieve the peptides with relatively higher antioxidant capability using hydroxyl radical- scavenging ability as response value. Then the antioxidant activity of obtained peptides was compared with POR.

\section{Materials and Methods}

\section{Materials}

Dried OR was purchased from Huangzhihua Pharmaceutical Co. Ltd (Jilin, Changchun, China) and authenticated by Prof. Limei Wang, School of Biology and Food Engineering, Changshu Institute of Technology (Jiangsu, Changshu, China). POR was prepared by ourselves according to previously published work (Zhang et al., 2019b).

Standards for the qualitative and quantitative analysis of amino acids were from Wako Pure Chemical Industries Ltd (Tokyo, Japan). Pepsin and trypsin were obtained from Aladdin Biochemical Technology Co., Ltd (Shanghai, China). Other reagents, such as salicylic acid, ferrous sulfate, 1, 1-diphenyl-2-picrylhydrazyl (DPPH) and vitamin C (VC) were obtained from Sigma Aldrich Chemical Co. Ltd (St. Louis, MO, USA).

Solvents were with analytical grade and provided by Sinopharm Chemical Reagent Co., Ltd (Shanghai, China).

\section{Extraction of Antioxidant Peptides from OR}

Before experiment, OR samples were pretreated by being grinded into powers and sieved to $100 \mathrm{mesh}$.
Approximately $0.6 \mathrm{~g}$ of OR powers were immersed in $30 \mathrm{~mL}$ of distilled water at room temperature overnight, then adjusted $\mathrm{pH}$ value to 1.2 using $1.0 \mathrm{~mol} / \mathrm{L}$ hydrochloric acid solution, followed by adding certain amount of pepsin (w/w) and being incubated at $37^{\circ} \mathrm{C}$ for certain time (h). After inactivating pepsin in boiling bath for $5 \mathrm{~min}$, $\mathrm{pH}$ value was adjusted into 8.0, then certain amount of trypsin (w/w) was added and incubated at $37^{\circ} \mathrm{C}$ for certain time (h). After inactivating trypsin in boiling bath for $5 \mathrm{~min}$, the mixture was centrifuged at $8000 \mathrm{r} / \mathrm{min}$ for $30 \mathrm{~min}$ at $4^{\circ} \mathrm{C}$, the obtained supernatant was lyophilized to give peptides, which were subsequently subjected to an evaluation of hydroxyl radical- scavenging capacity at the concentration of 5.0 $\mathrm{mg} / \mathrm{mL}$ (Zheng et al., 2019; Zhang et al., 2019b).

\section{Single-Factor Experiment}

Four factors that significantly affect the antioxidant ability of peptides from OR, including amount of pepsin $(1.0 \sim 3 \%$, equivalent to the weight percentage of OR powers), time of pepsin hydrolysis $(1.5 \mathrm{~h} \sim 4.0$ h), the amount of trypsin $(1.0 \sim 3.0 \%$, equivalent to the weight percentage of OR powers) and time of trypsin hydrolysis $(1.5 \mathrm{~h} \sim 4.0 \mathrm{~h})$ were selected to achieve the variables for process optimization.

\section{Response Surface Methodology Optimization}

Combined with the results of single-factor tests, a four-factor-three-level Response Surface Methodology (RSM) was achieved by Box-Behnken Design (BBD), which was shown in Table 1 (Zhang et al., 2019b).

Regression analysis was performed and fitted to the following second-order polynomial model:

$$
Y=\beta_{O}+\sum_{i=1}^{4} \beta_{i} X_{i}+\sum_{i=1}^{4} \beta_{i i} X_{i}^{2}+\sum_{i=1}^{3} \sum_{j=i+1}^{4} \beta_{i j} X_{i} Y_{j}
$$

Where,

$$
\begin{array}{ll}
Y & =\text { Response value } \\
\beta_{0} & =\text { Intercept } \\
\beta_{i} & =\text { Linear coefficient } \\
\beta_{i i} & =\text { Quadratic coefficient } \\
\beta_{i j} & =\text { Interactive term } \\
X_{i} \text { and } X_{j} & =\text { Independent variables }
\end{array}
$$

RSM was designed and analyzed with a Design-Expert software 8.0.6.1 (Stat-Ease, Minneapolis, MN, USA).

\section{Amino Acid Composition of Antioxidant Peptides from $O R$}

Antioxidant Peptides from OR (APOR) prepared by the optimal process were selected for further amino acid analysis. Fifty milliliters of APOR were accurately weighed and mixed with $5 \mathrm{~mL}$ of $6 \mathrm{moL} / \mathrm{L}$ hydrochloric acid solution at $110^{\circ} \mathrm{C}$ for $24 \mathrm{~h}$. When reaction completed, $4 \mathrm{~mol} / \mathrm{L}$ lithium hydroxide solution was added to adjust 
$\mathrm{pH}$ to 2.2 , then a fully automated amino acid analyzer (L-8900 Hitachi, Tokyo, Japan) was used to analyze the amino acids composition. Analytical conditions were: Cation exchange chromatographic column $(4.6 \mathrm{~mm} \times$ $60 \mathrm{~mm}$ ); column oven temperature of $55^{\circ} \mathrm{C}$; mobile phase of citric acid-sodium citrate at flow rate of 0.2 $\mathrm{mL} / \mathrm{min}$; chromogenic agent of ninhydrin solution at a flow rate of $0.12 \mathrm{~mL} / \mathrm{min}$; derivatization temperature of $135^{\circ} \mathrm{C}$; wavelength of 570 and $440 \mathrm{~nm}$, respectively; injection volume of $20 \mu \mathrm{L}$ (Zhang et al., 2019b).

\section{Hydroxyl Radical-Scavenging Activity}

APOR that had been obtained by the optimal conditions were made into distilled water solutions with concentration range of $2.0 \sim 10 \mathrm{mg} \cdot \mathrm{mL}^{-1}$. Two milliliters of APOR solution and $1 \mathrm{~mL}$ of PBS solution containing $0.6 \mathrm{mmol} / \mathrm{L} \mathrm{1,} \mathrm{10-phenanthroline}(\mathrm{pH} 7.4)$ were mixed, then $1 \mathrm{~mL}$ of $0.6 \mathrm{mmol} / \mathrm{L}$ ferrous sulfate solution and $1 \mathrm{~mL}$ of hydrogen peroxide solution $(0.2 \%, \mathrm{v} / \mathrm{v})$ were added. After incubation at $37^{\circ} \mathrm{C}$ for 1 $\mathrm{h}$, the absorbance at $536 \mathrm{~nm}\left(A_{s}\right)$ was read. The reaction system in absence of hydrogen peroxide was served as normal control $\left(A_{c}\right)$ and reaction system without APOR was used as blank control $\left(\mathrm{A}_{0}\right)$. Vitamin $\mathrm{C}(\mathrm{VC})$ was chosen as positive control. The hydroxyl radical-scavenging rate was deemed as the following formula (Zhang et al., 2019b):

$$
\begin{aligned}
& \text { Hydroxyl radical - scavenging rate }(\%) \\
& =\left(A_{s}-A_{0}\right) /\left(A_{c}-A_{0}\right) \times 100
\end{aligned}
$$

\section{Statistical Analysis}

Results were represented as the means \pm SD (standard deviation). RSM optimization was conducted by using Design-Expert software 8.0.6.1 (Stat-Ease, Minneapolis, MN, USA). The $t$-test and one-way analysis of variance (ANOVA) were performed for evaluating the significance, respectively.

\section{Results}

\section{Single-Factor Experiment}

Effects of Pepsin Amount on Hydroxyl RadicalScavenging Activity

It can be seen from Fig. 1 that when pepsin amount ranged from 1.0 to $2.0 \%$, the hydroxyl radicalscavenging rate of APOR increased with the increase of pepsin amount. However, when pepsin amount exceeded $2.0 \%$, the hydroxyl radical-scavenging rate decreased with the increase of pepsin amount. Therefore, $2.0 \%$ was selected as the optimal amount of pepsin.
Effects of Pepsin Incubation Time on Hydroxyl Radical-Scavenging Rate

As shown in Fig. 2, in the range of $1.5 \mathrm{~h} \sim 3.5 \mathrm{~h}$, the hydroxyl-radical scavenging rate of APOR increased with the extension of pepsin incubation time. When pepsin incubation time reached $3.5 \mathrm{~h}$, after that, the hydroxyl-radical scavenging rate was reduced with the prolongation of pepsin incubation time. Thus $3.5 \mathrm{~h}$ was chosen as the optimal pepsin incubation time.

Effects of Trypsin Amount on Hydroxyl RadicalScavenging Rate

As shown in Fig. 3, when trypsin amount spanned from 1.0 to $2.0 \%$, the hydroxyl radical-scavenging rate of APOR increased with the increase of trypsin amount. However, when trypsin amount surpassed $2.0 \%$, the hydroxyl radical-scavenging rate was reduced with the elevation of trypsin amount. Hence, $2.0 \%$ was elected as the optimal amount of trypsin.

\section{Effects of Trypsin Incubation Time on Hydroxyl Radical-Scavenging Rate}

From Fig. 4, it can be seen that the hydroxyl radicalscavenging rate of APOR increased with the increase of trypsin incubation time in the range of $1.5 \mathrm{~h} \sim 3.0 \mathrm{~h}$. When trypsin incubation time exceeded $3.0 \mathrm{~h}$, the hydroxyl radical-scavenging rate decreased with the extension of trypsin incubation time. Therefore, $3.0 \mathrm{~h}$ was selected as the optimal incubation time for trypsin.

\section{Response Surface Methodology Optimization}

\section{Programs and Results}

By using Design-Expert software 8.0.6.1, based on BBD, a four-factor-three-level RSM scheme was achieved in Table 2 .

\section{Model Establishment and Significance Test}

Via multiple regression fitting, the quadratic regression model equation was obtained as follows:

$$
\begin{aligned}
& Y=76.48+1.25 X_{1}+1.32 X_{2}-0.5 X_{3}-0.53 X_{4}-0.72 X_{1} X_{2}+ \\
& 1.83 X_{1} X_{3}-3.04 X_{1} X_{4}-3.61 X_{2} X_{3}-6.44 X_{2} X_{4}+4.2 X_{3} X_{4}- \\
& 8.64 X_{1}^{2}-14.21 X_{2}^{2}-9.54 X_{3}^{2}-14.46 X_{4}^{2}
\end{aligned}
$$

Where,

$Y=$ Hydroxyl radical-scavenging rate $(\%)$

$X_{1}=$ Amount of pepsin $(\%, \mathrm{w} / \mathrm{w})$

$X_{2}=$ Pepsin incubation time $(\mathrm{h})$

$X_{3}=$ Amount of trypsin $(\%, \mathrm{w} / \mathrm{w})$

$X_{4}=$ Trypsin incubation time $(\mathrm{h})$

One-way analysis of variance (ANOVA) for fitting equation was described in Table 3 . 


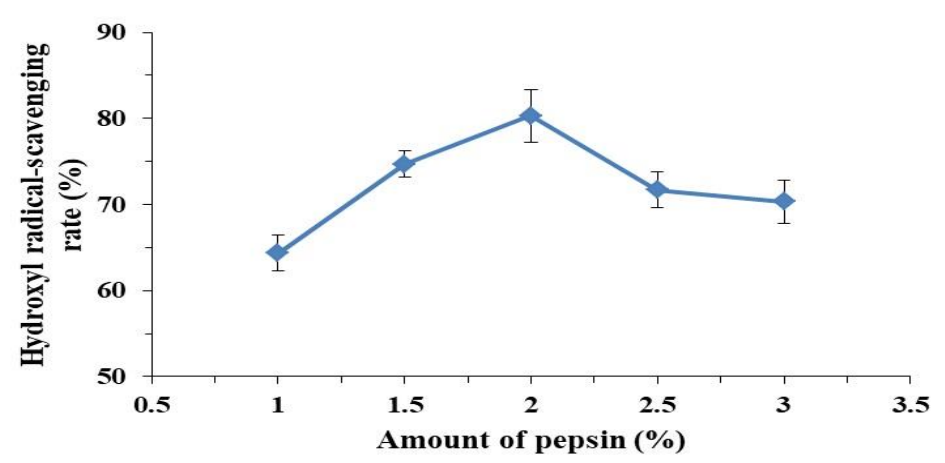

Fig. 1: Effects of pepsin amount on hydroxyl radical-scavenging rate

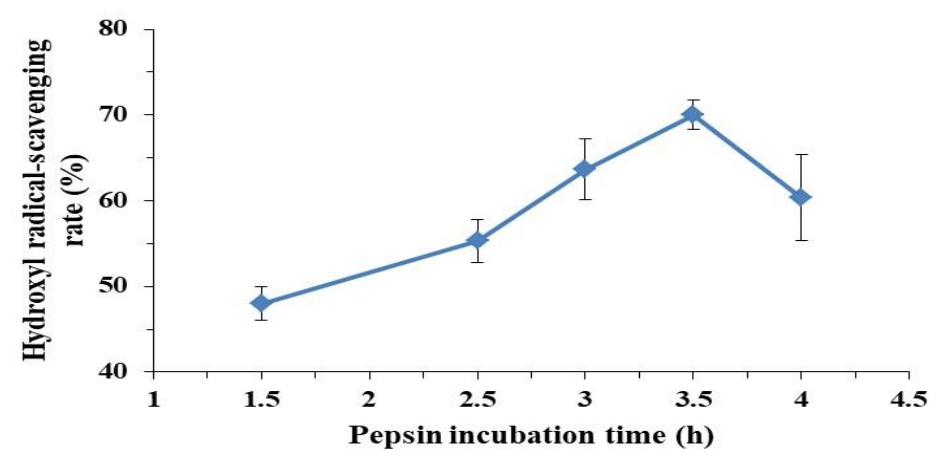

Fig. 2: Effects of pepsin incubation time on hydroxyl radical-scavenging rate

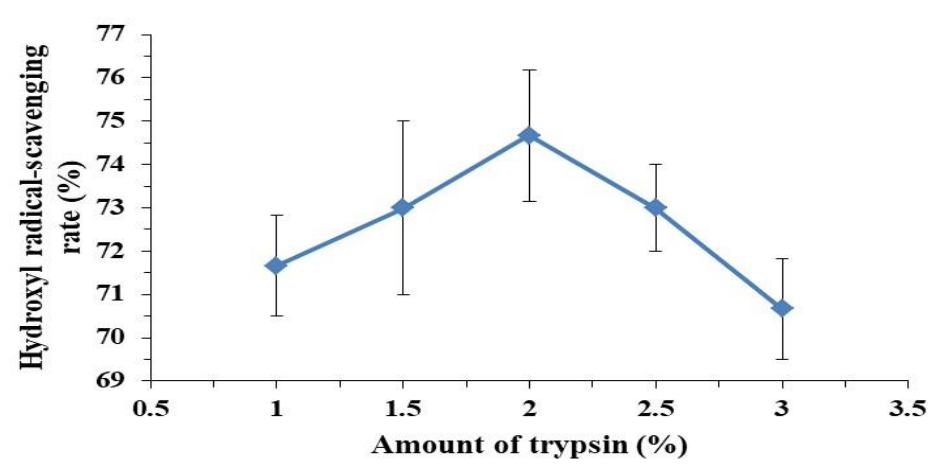

Fig. 3: Effects of trypsin amount on hydroxyl radical-scavenging rate

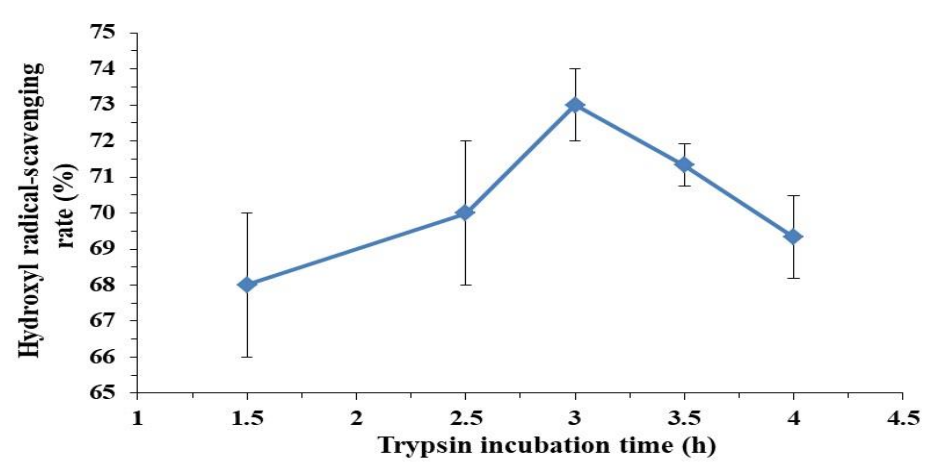

Fig. 4: Effects of trypsin incubation time on hydroxyl radical-scavenging rate 
From Table 3 , it can be observed that the $P$ value of model was $<0.0001 \quad(P<0.01)$ and the $P$ value of lack of fit was $0.3499(P>0.05)$, indicating that $(1)$ the fitting degree of this model is good; (2) unknown factors exert little influence on the results; (3) this model is stable and can well predict the actual changes of hydroxyl radical- scavenging rate of APOR (Kong et al., 2021). Coefficient of determination $\left(R^{2}\right)$ was 0.9288, suggesting that $92.88 \%$ of the changes in response values derive from the selected variables. The adjusted $\mathrm{R}^{2}$ was 0.8576 , implying that variabilities of $85.76 \%$ of the test data can be explained by this model.

\section{Graphical Analysis}

The graph of RSM is a three-dimensional space map composed of specific response values corresponding to independent variables. It can directly reflect the influence of their respective variables on the response values. According to regression model, response surface and the corresponding contour map can be obtained, respectively (Wei et al., 2019).

Interaction Effects of Pepsin Amount $\left(X_{1}\right)$ and Pepsin Incubation Time $\left(X_{2}\right)$ on Hydroxyl RadicalScavenging Rate

As shown in Fig. 5, when pepsin amount was in the range of $1.5 \sim 2.5 \%$, the hydroxyl radical-scavenging rate of APOR increased at first and then decreased with the increase of pepsin amount, which was consistent with the results of single-factor experiment. Similarly, the hydroxyl radical-scavenging rate of APOR increased at first and then decreased with the extension of pepsin incubation time, but the change trend was slow. The effects of pepsin amount on hydroxyl radical-scavenging rate was greater than that of pepsin incubation time and the interaction between pepsin incubation time and pepsin amount was not significant.

Interaction Effects of Pepsin Amount $\left(X_{1}\right)$ and Trypsin Amount $\left(X_{3}\right)$ on Hydroxyl RadicalScavenging Rate

Figure 6 , it can be seen that the hydroxyl radicalscavenging rate of APOR increased with pepsin amount ranging from $1.5 \sim 2.5 \%$, then decreased with the increase of pepsin amount and the trend of changes was relatively gentle. Within the range of $1.5 \sim 2.5 \%$ trypsin amount and the overall changes of hydroxyl radical-scavenging rate were slow and the interaction between pepsin amount and trypsin amount was not significant.

Interaction Effects of Pepsin Amount $\left(X_{1}\right)$ and Trypsin Incubation Time $\left(X_{4}\right)$ on Hydroxyl RadicalScavenging Rate

Figure 7 , within the range of $1.5 \sim 2.5 \%$ pepsin amount, the hydroxyl radical-scavenging rate of APOR increased at first and then decreased with the changes of pepsin amount and the trend of changes was relatively gentle. In the range of trypsin incubation time $2.5 \mathrm{~h} \sim 3.5 \mathrm{~h}$, the overall changes of hydroxyl radical- scavenging rate were slow and the interaction between pepsin amount and trypsin incubation time was not significant.

Interaction Effects of Pepsin Incubation Time $\left(X_{2}\right)$ and Trypsin Amount $\left(X_{3}\right)$ on Hydroxyl RadicalScavenging Rate

From Fig. 8, it can be observed that when pepsin incubation time was in the range of $3.0 \mathrm{~h} \sim 4.0 \mathrm{~h}$, the hydroxyl radical-scavenging rate of APOR increased at first and then decreased with the changes of trypsin amount. Within the range of $1.5 \sim 2.5 \%$ trypsin amount, the hydroxyl radical scavenging rate increased continuously, when reaching the summit, the hydroxyl radical-scavenging rate decreased slightly with the increase of pepsin incubation time, indicating that the interaction between pepsin incubation time and trypsin amount was significant.

Interaction Effects of Pepsin Incubation Time $\left(X_{2}\right)$ and Trypsin Incubation Time $\left(X_{4}\right)$ on Hydroxyl Radical-Scavenging Rate Radical-Scavenging Rate

As shown in Fig. 9, when pepsin incubation time was within the range of $3.0 \mathrm{~h} \sim 4.0 \mathrm{~h}$, the hydroxyl radicalscavenging rate of APOR increased at first and then decreased with the changes of trypsin incubation time. In the range of trypsin incubation time $2.5 \mathrm{~h} \sim 3.5 \mathrm{~h}$, the hydroxyl radical scavenging rate increased continuously, after reaching the highest value, the hydroxyl radical- scavenging rate decreased obviously with the increase of pepsin incubation time, indicating that the interaction between pepsin incubation time and trypsin incubation time was highly significant.

Interaction Effects of Trypsin Amount $\left(X_{3}\right)$ and Trypsin Incubation Time $\left(X_{4}\right)$ on Hydroxyl RadicalScavenging Rate

Figure 10, when trypsin amount was within the range of $1.5 \sim 2.5 \%$, the hydroxyl radical- scavenging rate increased at first and then decreased with the changes of trypsin amount. In the range of trypsin incubation time $2.5 \mathrm{~h} \sim 3.5 \mathrm{~h}$, the hydroxyl radicalscavenging rate increased continuously, after reaching the summit, the hydroxyl radical-scavenging rate decreased with the increase of trypsin amount, indicating that the interaction between trypsin amount and trypsin incubation time was slightly significant. 

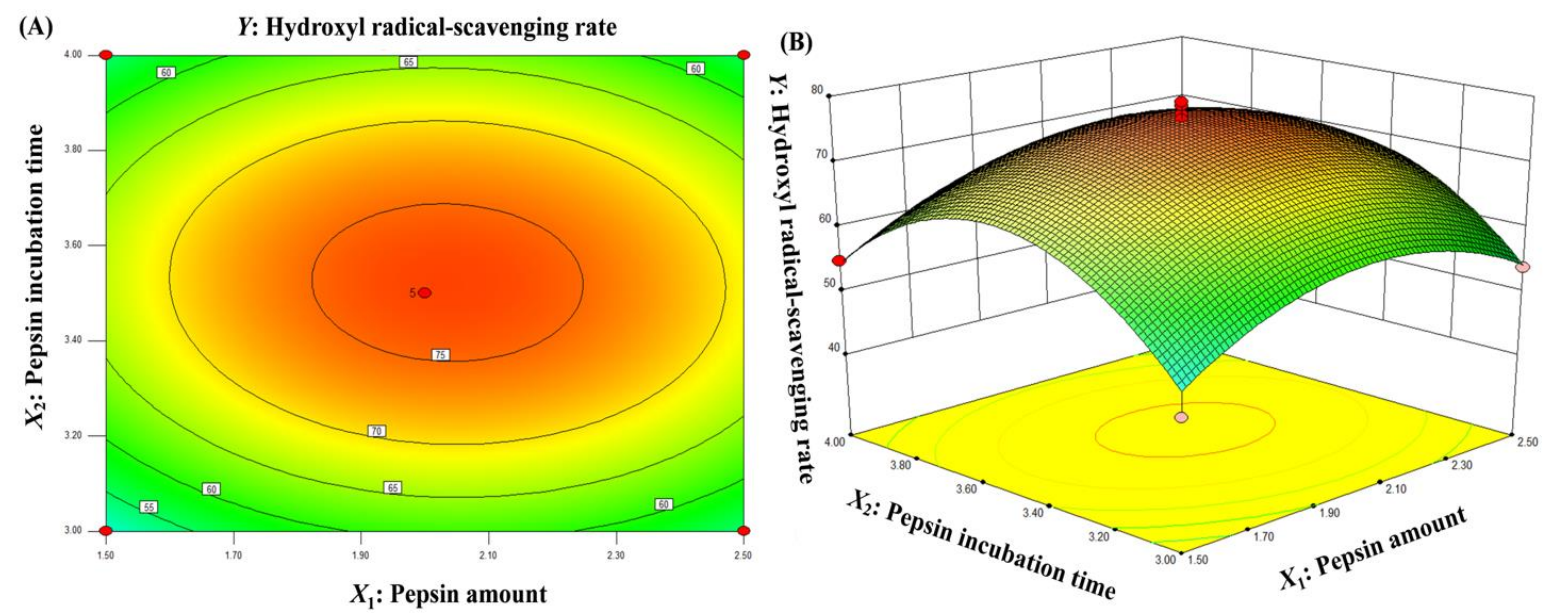

Fig. 5: Contour plot (A) and response surface (B) that display the interaction effects of pepsin amount and pepsin incubation time on hydroxyl radical-scavenging rate
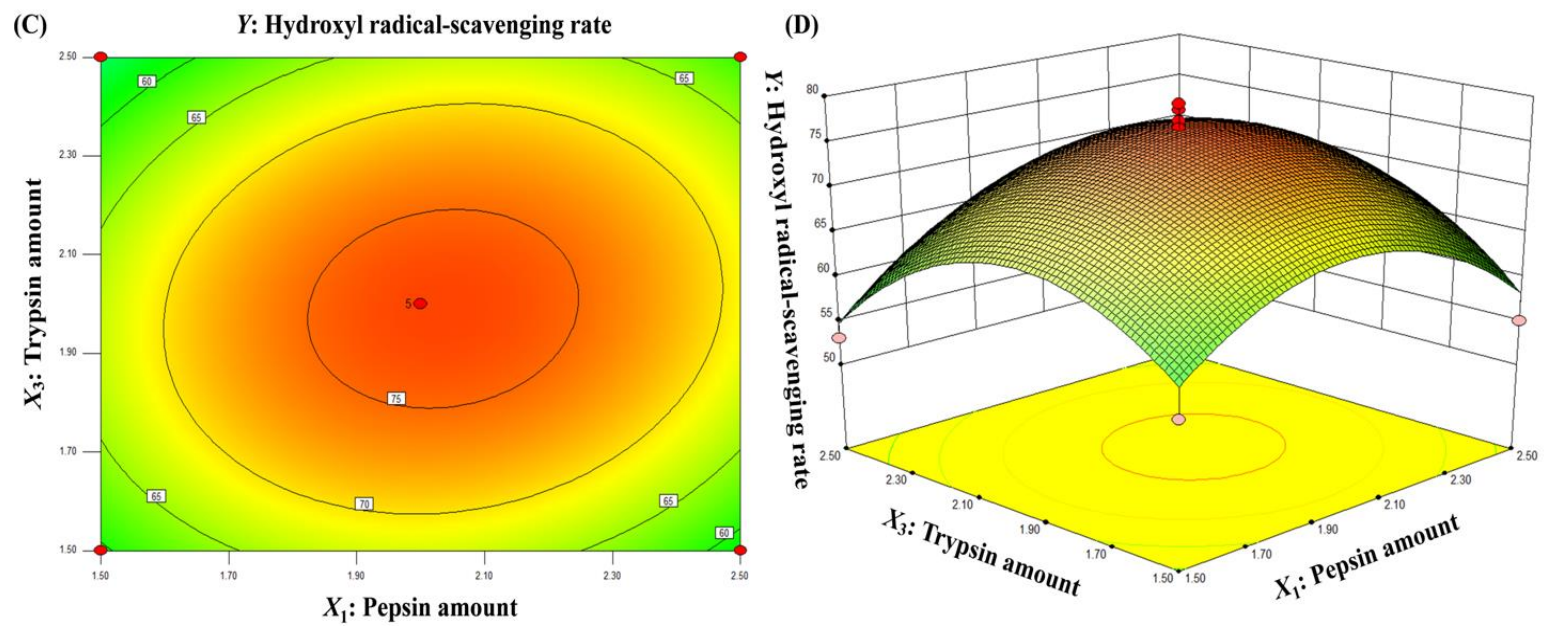

Fig. 6: Contour plot (C) and response surface (D) that display the interaction effects of pepsin amount and trypsin amount on hydroxyl radical-scavenging rate
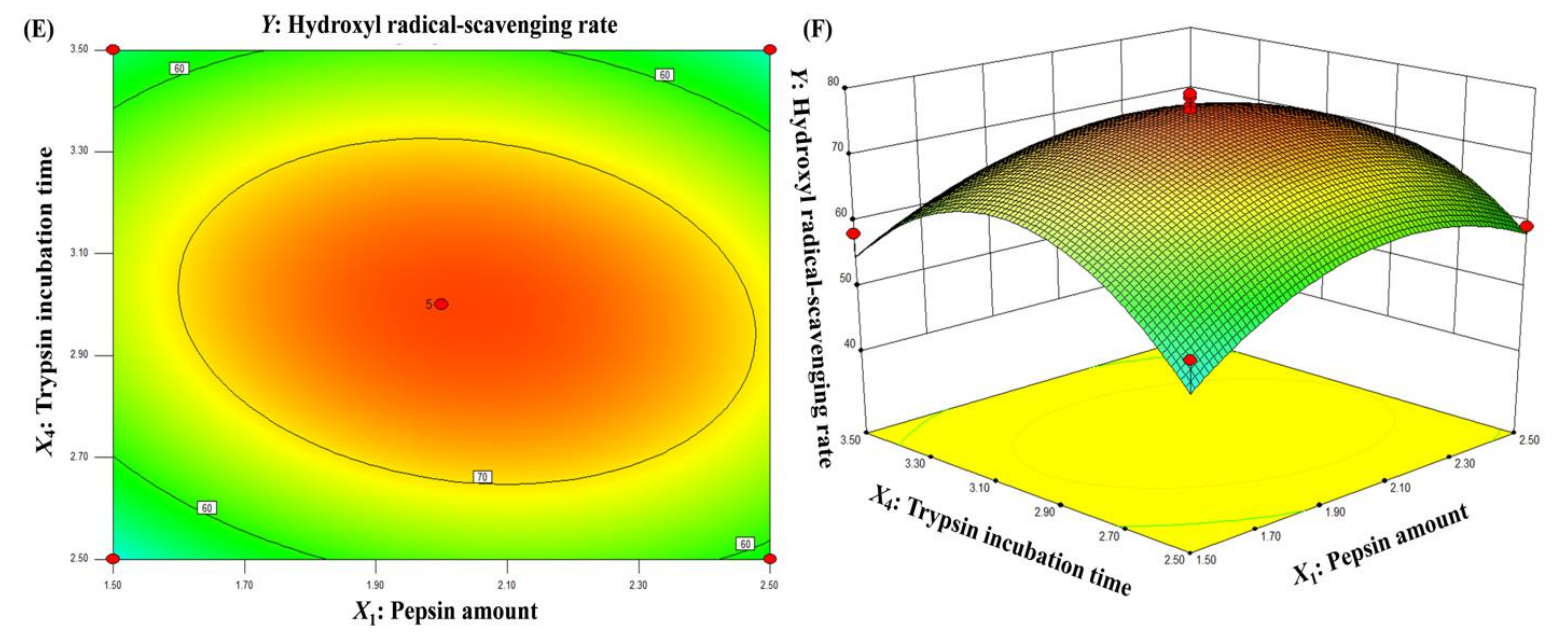

Fig. 7: Contour plot (E) and response surface $(F)$ that display the interaction effects of pepsin amount and trypsin incubation time on hydroxyl radical-scavenging rate 

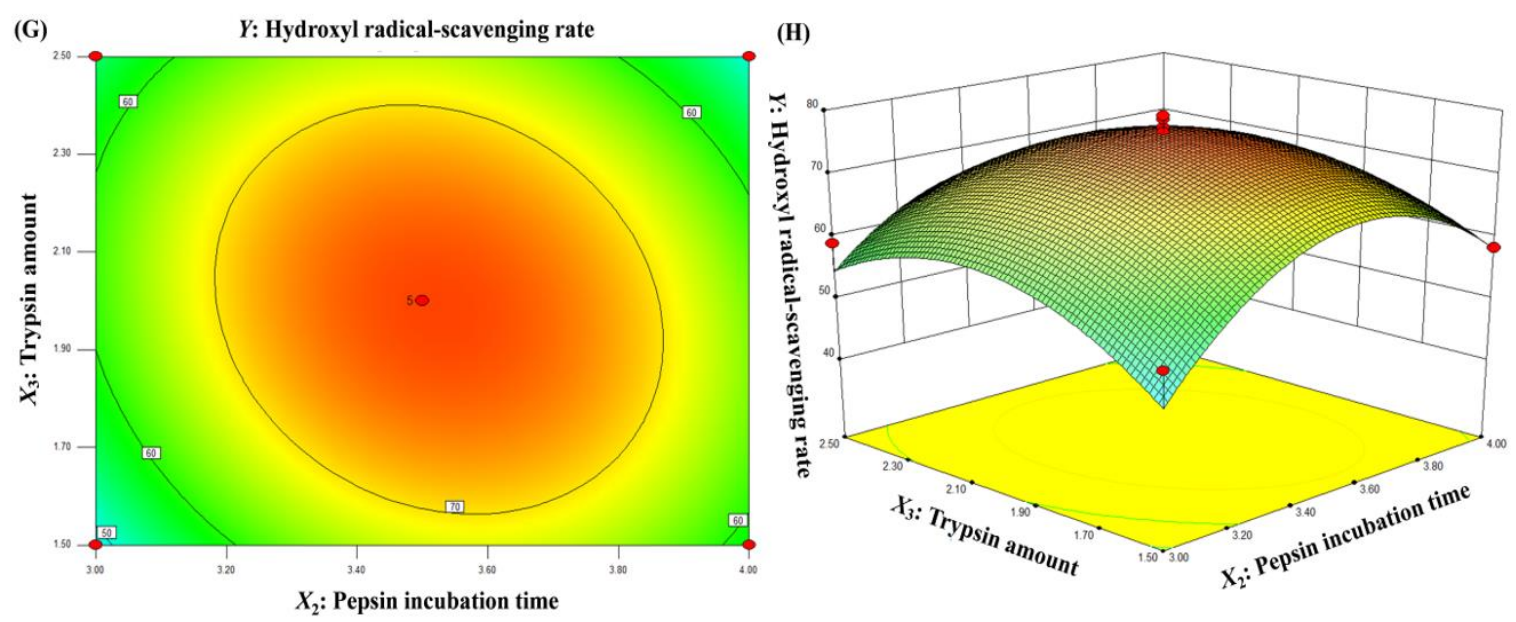

Fig. 8: Contour plot $(G)$ and response surface $(H)$ that display the interaction effects of pepsin incubation time and trypsin amount on hydroxyl radical-scavenging rate
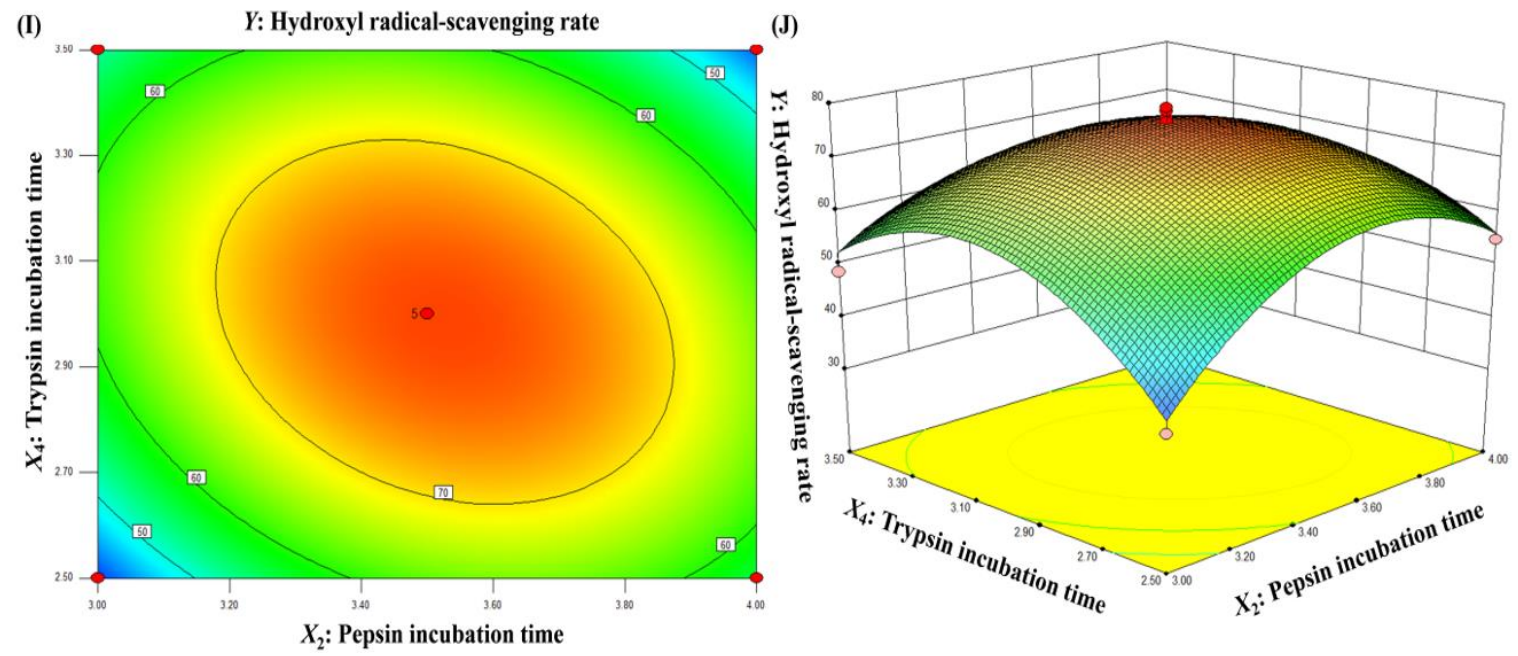

Fig. 9: Contour plot (I) and response surface (J) that display the interaction effects of pepsin incubation time and trypsin incubation time on hydroxyl radical-scavenging rate
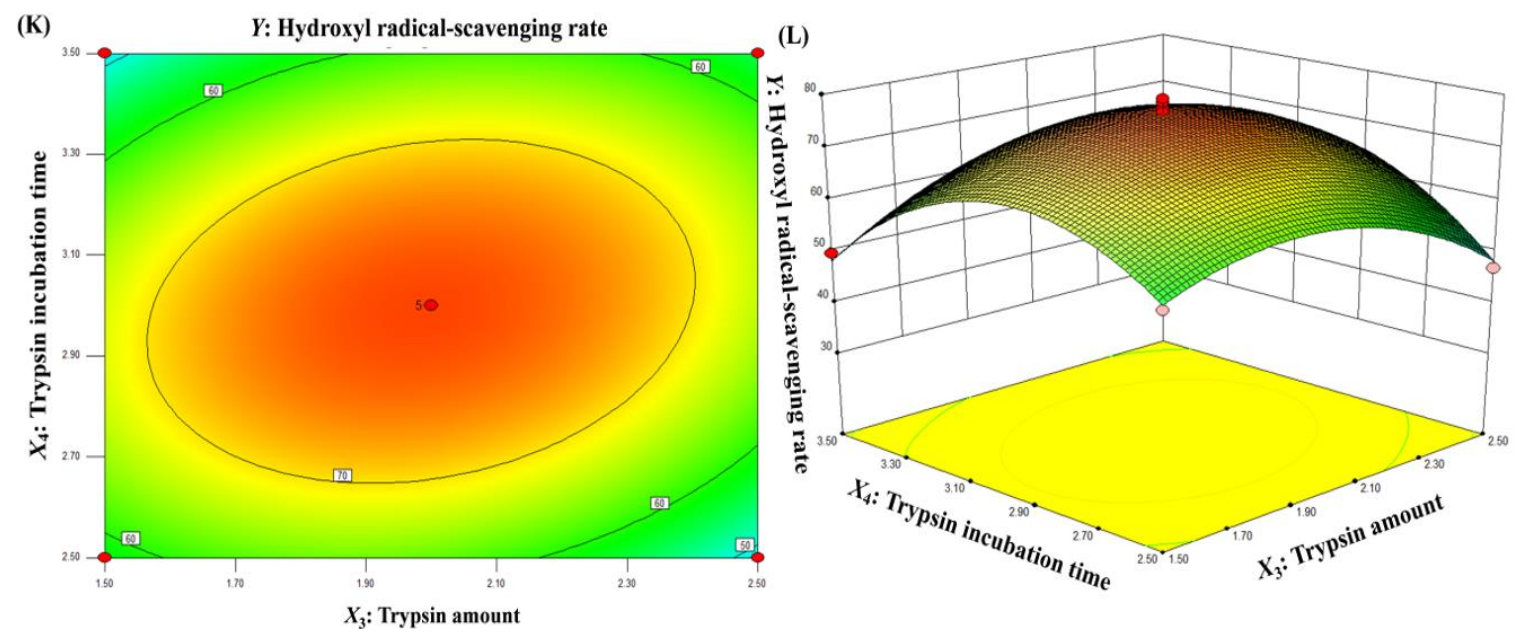

Fig. 10: Contour plot $(\mathrm{K})$ and response surface $(\mathrm{L})$ that display the interaction effects of trypsin amount and trypsin incubation time on hydroxyl radical-scavenging rate 


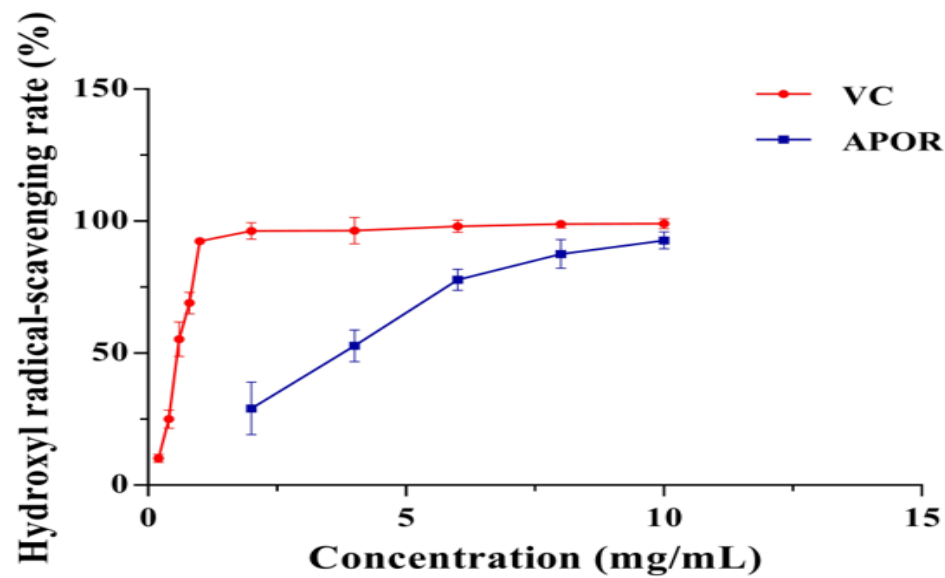

Fig. 11: Hydroxyl radical-scavenging activity of APOR using VC as positive control. Data was represented as the mean \pm SD $(n=3)$

Table 1: Variables and levels for response surface methodology optimization

\begin{tabular}{llll}
\hline & Coding levels & & \\
Variables & -1 & 0 & +1 \\
\hline Amount of pepsin $\left(\%, w / w, X_{1}\right)$ & 1.5 & 3.5 & 2.5 \\
Pepsin incubation time $\left(\mathrm{h}, X_{2}\right)$ & 3.0 & 2.0 & 4.0 \\
Amount of trypsin $\left(\%, \mathrm{w} / \mathrm{w}, X_{3}\right)$ & 1.5 & 3.0 & 2.5 \\
Trypsin incubation time $\left(\mathrm{h}, X_{4}\right)$ & 2.5 & 3.5 \\
\hline
\end{tabular}

Table 2: Programs and results of RSM experiment

\begin{tabular}{|c|c|c|c|c|c|}
\hline \multirow[b]{2}{*}{ Run } & \multicolumn{4}{|c|}{ Independent variables } & \multirow[b]{2}{*}{$Y / \%$} \\
\hline & $X_{1} / \%$ & $X_{2} / \mathrm{h}$ & $X_{3} / \%$ & $X_{4} / \mathrm{h}$ & \\
\hline 1 & 2.00 & 3.50 & 2.00 & 3.00 & 76.56 \\
\hline 2 & 2.00 & 3.50 & 2.50 & 2.50 & 46.86 \\
\hline 3 & 2.50 & 3.50 & 1.50 & 3.00 & 55.07 \\
\hline 4 & 2.50 & 3.50 & 2.00 & 3.50 & 51.18 \\
\hline 5 & 2.50 & 3.50 & 2.50 & 3.00 & 59.39 \\
\hline 6 & 1.50 & 3.50 & 2.50 & 3.00 & 53.06 \\
\hline 7 & 2.00 & 3.50 & 1.50 & 3.50 & 49.78 \\
\hline 8 & 2.00 & 3.50 & 2.00 & 3.00 & 79.18 \\
\hline 9 & 2.00 & 3.50 & 2.00 & 3.00 & 77.32 \\
\hline 10 & 2.00 & 4.00 & 2.50 & 3.00 & 49.11 \\
\hline 11 & 2.00 & 3.00 & 2.00 & 2.50 & 38.30 \\
\hline 12 & 1.50 & 4.00 & 2.00 & 3.00 & 54.91 \\
\hline 13 & 2.50 & 4.00 & 2.00 & 3.00 & 59.33 \\
\hline 14 & 1.50 & 3.00 & 2.00 & 3.00 & 46.58 \\
\hline 15 & 2.00 & 3.50 & 2.50 & 3.50 & 56.59 \\
\hline 16 & 2.50 & 3.00 & 2.00 & 3.00 & 53.86 \\
\hline 17 & 2.00 & 4.00 & 2.00 & 3.50 & 39.63 \\
\hline 18 & 2.00 & 3.00 & 1.50 & 3.00 & 53.91 \\
\hline 19 & 1.50 & 3.50 & 1.50 & 3.00 & 56.08 \\
\hline 20 & 1.50 & 3.50 & 2.00 & 2.50 & 54.27 \\
\hline 21 & 2.00 & 3.00 & 2.00 & 3.50 & 48.78 \\
\hline 22 & 2.00 & 3.50 & 2.00 & 3.00 & 78.54 \\
\hline 23 & 1.50 & 3.50 & 2.00 & 3.50 & 58.27 \\
\hline 24 & 2.00 & 3.00 & 2.50 & 3.00 & 59.04 \\
\hline 25 & 2.50 & 3.50 & 2.00 & 2.50 & 59.36 \\
\hline 26 & 2.00 & 4.00 & 2.00 & 2.50 & 54.92 \\
\hline 27 & 2.00 & 3.50 & 2.00 & 3.00 & 70.79 \\
\hline 28 & 2.00 & 4.00 & 1.50 & 3.00 & 58.40 \\
\hline 29 & 2.00 & 3.50 & 1.50 & 2.50 & 56.84 \\
\hline
\end{tabular}


Table 3: ANOVA for response surface quadratic model ${ }^{\mathrm{a}}$

\begin{tabular}{|c|c|c|c|c|c|c|}
\hline Source $^{b}$ & Sum of squares & Freedom & Mean square & $F$-value & $P$-value & Significance $^{c}$ \\
\hline Model & 2880.6 & 14 & 205.76 & 13.05 & $<0.0001$ & \\
\hline$X_{1}$ & 18.8 & 1 & 18.80 & 1.19 & 0.2933 & n.s. \\
\hline$X_{2}$ & 20.88 & 1 & 20.88 & 1.32 & 0.2691 & n.s. \\
\hline$X_{3}$ & 3.03 & 1 & 3.03 & 0.19 & 0.6678 & n.s. \\
\hline$X_{4}$ & 3.33 & 1 & 3.33 & 0.21 & 0.6530 & n.s. \\
\hline$X_{1} X_{2}$ & 2.04 & 1 & 2.04 & 0.13 & 0.7241 & n.s. \\
\hline$X_{1} X_{3}$ & 13.47 & 1 & 13.47 & 0.85 & 0.3711 & n.s. \\
\hline$X_{1} X_{4}$ & 37.09 & 1 & 37.09 & 2.35 & 0.1474 & n.s. \\
\hline$X_{2} X_{3}$ & 51.98 & 1 & 51.98 & 3.30 & 0.0209 & $*$ \\
\hline$X_{2} X_{4}$ & 166.02 & 1 & 166.06 & 10.53 & 0.0009 & ** \\
\hline$X_{3} X_{4}$ & 70.48 & 1 & 70.48 & 4.47 & 0.0429 & * \\
\hline$X_{1}^{2}$ & 483.69 & 1 & 483.09 & 30.67 & $<0.0001$ & $* *$ \\
\hline$X_{2}^{2}$ & 1309.61 & 1 & 1309.61 & 37.43 & $<0.0001$ & $* *$ \\
\hline$X_{3}^{2}$ & 590.23 & 1 & 590.23 & 85.98 & $<0.0001$ & ** \\
\hline$X_{4}^{2}$ & 1355.86 & 1 & 1355.86 & & $<0.0001$ & ** \\
\hline Residual & 220.78 & 14 & 15.77 & & & \\
\hline Lack of fit & 176.15 & 10 & 17.62 & 1.58 & 0.3499 & n.s. \\
\hline Cor total & 3101.37 & 28 & & & & \\
\hline R-Squared & 0.9288 & & & & & \\
\hline Adj R-Squared & 0.8576 & & & & & \\
\hline
\end{tabular}

Table 4: Amino acids composition of APOR prepared by the optimal parameters

\begin{tabular}{llll}
\hline No. & Amino acids & Content $/{\mathrm{mg} . \mathrm{g}^{-1}}^{-1}$ & Percentage/\% \\
\hline 1 & a'Histidine & 70.9 & 13.8 \\
2 & Cystine & 69.4 & 13.5 \\
3 & Proline & 68.6 & 13.3 \\
4 & a'Threonine & 50.4 & 9.4 \\
5 & Aspartate & 42.5 & 7.7 \\
6 & Glutamate & 38.5 & 6.8 \\
7 & Serine & 32.6 & 5.5 \\
8 & a'Lysine & 31.7 & 5.3 \\
9 & Glycine & 25.2 & 3.9 \\
10 & aLeucine & 23.9 & 3.7 \\
11 & aValine & 22.0 & 3.3 \\
12 & asoleucine & 21.2 & 3.1 \\
13 & Tyrosine & 19.6 & 2.7 \\
14 & Arginine & 19.5 & 2.7 \\
15 & aPhenylalanine & 18.6 & 2.5 \\
16 & Alanine & 16.3 & 2.0 \\
17 & aMethionine & 10.4 & 0.7 \\
-- & Total & 581.3 & 100 \\
\hline
\end{tabular}

\section{Verification of Predictive Model}

The optimal conditions for APOR preparation obtained from Design-Expert software were: Pepsin amount of $2.04 \%$, pepsin incubation time of $3.53 \mathrm{~h}$, trypsin amount of $1.98 \%$ and trypsin incubation time of $2.98 \mathrm{~h}$. Under these conditions, the theoretical hydroxyl radical-scavenging rate of APOR was $76.5917 \%$. In order to test the reliability of the results obtained by RSM, the above-mentioned optimal conditions were used to prepare APOR in practice, considering the convenience of operation, parameters were modified as follows: Pepsin amount of $2.0 \%$, pepsin incubation time of $3.5 \mathrm{~h}$, trypsin amount of $2.0 \%$ and trypsin incubation time of $3.0 \mathrm{~h}$, under these conditions, the actual hydroxyl radical-scavenging rate of APOR was $75.52 \pm 1.92 \%$ and extraction yield was $63.45 \pm 1.43 \%$. Compared with theoretical prediction, the relative error was $\pm 1.42 \%$.

\section{Amino Acids Composition}

The amino acids of APOR that had been obtained under the optimal conditions were subsequently determined. As shown in Table 4, there were 17 amino acids present in APOR, the total content of which was $581.3 \mathrm{mg} \cdot \mathrm{g}^{-1}$. Seven essential amino acids with total percentage of $41.8 \%$ were found in APOR. The top three amino acids were histidine, cystine and proline with histidine the highest level of $70.9 \mathrm{mg} \cdot \mathrm{g}^{-1}$. 


\section{Verification of Hydroxyl Radical-Scavenging Activity}

In order to further confirm the hydroxyl radical-scavenging contribution of APOR obtained under the optimal conditions, the hydroxyl radical-scavenging rates at different concentrations were evaluated using Vitamin C (VC) as positive control. From Fig. 11, in the concentration range of $2.0 \mathrm{mg} \cdot \mathrm{mL}^{-1} \sim 10 \mathrm{mg} \cdot \mathrm{mL}^{-1}$, the hydroxyl radical-scavenging rate of APOR increased with the increase of concentration $\left(\mathrm{y}=7.77 \mathrm{x}+20.727, \mathrm{R}^{2}=\right.$ $0.9008)$ and the calculated half inhibitory concentration $\left(\mathrm{IC}_{50}\right)$ was $3.71 \pm 0.71 \mathrm{mg} \cdot \mathrm{mL}^{-1}$. However, the $\mathrm{IC}_{50}$ value of $\mathrm{VC}$ against hydroxyl radical was $0.60 \pm 0.02 \mathrm{mg} \cdot \mathrm{mL}^{-1}$, which was significantly lower $(P<0.01)$ than that of APOR.

\section{Discussion}

In present investigation, to imitate the in vivo digestive process of proteins, the antioxidant peptides from OR (APOR) were extracted by pepsin/trypsin-based stepwise hydrolysis. In single-factor experiment, the hydroxyl radical-scavenging rate of APOR was at first elevated with the increase of enzyme amount and incubation time, then decreased with the further increase of enzyme amount and incubation time (Figs $1 \sim 4$ ), which could be attributed to the saturability and negative feedback of enzymes (Yin et al., 2018). Afterwards, RSM was applied to optimize the extraction process by using BBD. Twenty-nine programs were given and the model used for predicting optimal parameters was reliable and feasible. From graphical analysis of contours and response surfaces, it can be concluded that the order of effects of variables on the hydroxyl radical-scavenging rate of AROP were: Trypsin incubation time > trypsin amount > pepsin incubation time $>$ pepsin amount (Fig. 5-10). After discussing the key factors that affect the hydroxyl radicalscavenging rate of AROP and their interactions, the optimal process parameters were obtained and listed as follows: Pepsin amount of $2.0 \%$, pepsin incubation time of $3.5 \mathrm{~h}$, trypsin amount of $2.0 \%$ and trypsin incubation time of $3.0 \mathrm{~h}$, under these conditions, the hydroxyl radicalscavenging rate of obtained APOR was $75.52 \pm 1.92 \%$, when compared with predicted value, the relative deviation was $\pm 1.42 \%$, less than $\pm 5.0 \%$, suggesting that RSM along with BBD would be a feasible way to optimize the extraction conditions for APOR (Kong et al., 2021).

APOR obtained by the above-mentioned optimal parameters comprise seventeen amino acids with total content of $581.3 \mathrm{mg} \cdot \mathrm{g}^{-1}$, which was higher than that of OR (426.8 mg.g ${ }^{-1}$ ) (Zhang et al., 2017), but lower than that of POR (668 mg.g ${ }^{-1}$ ) (Zhang et al., 2019b). These results indicated that enzymolysis could promote the release of amino acids from OR powders, leading to a relatively higher amino acids content of APOR than that of untreated counterpart. However, the amino acids yield of
APOR was smaller than that of POR, which could be due to the fact that some non-protein ingredients may hinder the release of amino acids from APOR. In the concentration range of $2.0 \mathrm{mg} \cdot \mathrm{mL}^{-1} \sim 10 \mathrm{mg} \cdot \mathrm{mL}^{-1}$, APOR exerted significant hydroxyl radical-scavenging capacity with $\mathrm{IC}_{50}$ value of $3.71 \pm 0.71 \mathrm{mg} . \mathrm{mL}^{-1}$, which was lower than that of POR $\left(4.85 \pm 0.06 \mathrm{mg} \cdot \mathrm{mL}^{-1}\right)$ (Zhang et al., 2019b), suggesting that the scavenging capacities of APOR against hydroxyl radicals were improved after pepsin/trypsin-mediated hydrolysis. The antioxidant activity of APOR could be partially contributed by its antioxidant amino acids. Previous studies have revealed that amino acids, including cysteine, threonine, tryptophan, methionine, tyrosine, phenylalanine, glutamate, aspartate, lysine and histidine exhibit excellent in vitro and in vivo antioxidant capacities (Zhang et al., 2019b). In APOR, there were eight antioxidant amino acids with total percentage of $48.9 \%$. Moreover, relatively lower molecular weights are more likely to make APOR more easily to interact with hydroxyl radicals (Wang et al., 2013), which deserves of deep exploration.

\section{Conclusion}

Pepsin/trypsin-based stepwise hydrolysis was used to obtain APOR, after process optimization with RSM, we concluded the optimal parameters. APOR prepared under the optimal conditions exerted higher hydroxyl radical-scavenging capacity than that of POR. The higher antioxidant activity could be contributed by the rich antioxidant amino acids and relatively lower molecular weights, which should be further verified in the near future. In addition, further purification, separation and characterization of APOR should be equally emphasized. As for industrial potential, present work preliminarily achieved a feasible way to obtain APOR using OR powders as starting materials at the laboratory level and pilot batch production will be still needed to be conducted collaborating with factories.

\section{Acknowledgement}

The authors express gratitude to Dr. Hongli Zhou, a professor at Jilin Institute of Chemical Technology, for her support.

\section{Funding Information}

This work was financed by the Program of Suzhou Science and Technology Bureau (Grant No. SNG201906), the Key Program of Jiangsu Provincial Undergraduate Innovation and Entrepreneurship Training in 2021 and the Programs of Changshu Institute of Technology. 


\section{Author's Contributions}

Jieyang Shen and Yihui Liu: Carried out the process optimization and wrote the paper.

Jiahao Xu and Shenbo Ye: Carried out the process optimization.

Xiaoxiao Xu: Performed the amino acids analysis.

Yang Liu: Performed the antioxidant assessment.

Lixue Zheng and Yang Zhang: Conceived the project, designed the experiments, revised and polished the manuscript.

\section{Ethics}

This article is original and contains unpublished material. The corresponding author confirms that all of the other authors have read and approved the manuscript and no ethical issues involved

\section{References}

Beverly, R. L., Huston, R. K., Markell, A. M., McCulley, E. A., Martin, R. L., \& Dallas, D. C. (2021). Differences in human milk peptide release along the gastrointestinal tract between preterm and term infants. Clinical Nutrition, 40(3), 1214-1223. doi.org/10.1016/j.clnu.2020.07.035

De Luca, C., Mikhal'chik, E. V., Suprun, M. V., Papacharalambous, M., Truhanov, A. I., \& Korkina, L. G. (2016). Skin antiageing and systemic redox effects of supplementation with marine collagen peptides and plant-derived antioxidants: A singleblind case-control clinical study. Oxidative medicine and cellular longevity, 2016. doi.org/10.1155/2016/4389410

Gorji, S. G., Calingacion, M., Smyth, H. E., \& Fitzgerald, M. (2019). Effect of natural antioxidants on lipid oxidation in mayonnaise compared with BHA, the industry standard. Metabolomics, 15(8), 1-14. doi.org/10.1007/s11306-019-1568-4

Hou, J. T., Zhang, M., Liu, Y., Ma, X., Duan, R., Cao, X., ... \& Ren, W. X. (2020). Fluorescent detectors for hydroxyl radical and their applications in bioimaging: A review. Coordination Chemistry Reviews, 421, 213457. doi.org/10.1016/j.ccr.2020.213457

Kong, Y., Wang, G., Wang, X., Wang, T., Shen, J., Zhang, A., ... \& Zhang, Y. (2021). Essential Oils from The Dropped Flowers of Camellia japonica: Extraction Optimization, Chemical Profile and Antibacterial Property. doi.org/10.3844/ajbbsp.2021.40.49

Kurniawan, H., Franchina, D. G., Guerra, L., Bonetti, L., Soriano-Baguet, L., Grusdat, M., ... \& Brenner, D. (2020). Glutathione restricts serine metabolism to preserve regulatory $\mathrm{T}$ cell function. Cell metabolism, 31(5), 920-936. doi.org/10.1016/j.cmet.2020.03.004
Martinez-Useros, J., Li, W., Cabeza-Morales, M., \& Garcia-Foncillas, J. (2017). Oxidative stress: A new target for pancreatic cancer prognosis and treatment. Journal of clinical medicine, 6(3), 29. doi.org/10.3390/jcm6030029

Schroeder, J., Alteheld, B., Stehle, P., Cayeux, M. C., Chiolero, R. L., \& Berger, M. M. (2005). Safety and intestinal tolerance of high-dose enteral antioxidants and glutamine peptides after upper gastrointestinal surgery. European journal of clinical nutrition, 59(2), 307-310. doi.org/10.1038/sj.ejcn.1602073

Wen, C., Zhang, J., Zhang, H., Duan, Y., \& Ma, H. (2020). Plant protein-derived antioxidant peptides: Isolation, identification, mechanism of action and application in food systems: A review. Trends in Food Science \& Technology. doi.org/10.1016/j.tifs.2020.09.019

Wei, E., Yang, R., Zhao, H., Wang, P., Zhao, S., Zhai, W., ... \& Zhou, H. (2019). Microwave-assisted extraction releases the antioxidant polysaccharides from seabuckthorn (Hippophae rhamnoides L.) berries. International journal of biological macromolecules, 123, 280-290. doi.org/10.1016/j.ijbiomac.2018.11.074

Wang, B., Li, L., Chi, C. F., Ma, J. H., Luo, H. Y., \& Xu, Y. F. (2013). Purification and characterisation of a novel antioxidant peptide derived from blue mussel (Mytilus edulis) protein hydrolysate. Food Chemistry, 138(2-3), 1713-1719. doi.org/10.1016/j.foodchem.2012.12.002

Xia, Y., Bamdad, F., Gänzle, M., \& Chen, L. (2012). Fractionation and characterization of antioxidant peptides derived from barley glutelin by enzymatic hydrolysis. Food Chemistry, 134(3), 1509-1518. doi.org/10.1016/j.foodchem.2012.03.063

Yan, W., Lin, G., Zhang, R., Liang, Z., \& Wu, W. (2020). Studies on the bioactivities and molecular mechanism of antioxidant peptides by 3D-QSAR, in vitro evaluation and molecular dynamic simulations. Food \& function, 11(4), 3043-3052. doi.org/10.1039/C9FO03018B

Yu, X. J., Wang, X.Y., Liu, X., Liu, L., \& Zhang, Y. (2020). A polysaccharide-rich fraction derived from Oviductus ranae with antioxidant and immunomodulatory potentials. American Journal of Biochemistry and Biotechnology, 16(3), 451-461. doi.org/10.3844/ajbbsp.2020.451.461

Yin, C., Fan, X., Fan, Z., Shi, D., \& Gao, H. (2018). Optimization of enzymes-microwave-ultrasound assisted extraction of Lentinus edodes polysaccharides and determination of its antioxidant activity. International journal of biological macromolecules, 111, 446-454. doi.org/10.1016/j.ijbiomac.2018.01.007

Zhang, Y., Wang, Y., Li, M., Liu, S., Yu, J., Yan, Z., \& Zhou, H. (2019a). Traditional uses, bioactive constituents, biological functions and safety properties of Oviductus ranae as functional foods in China. Oxidative medicine and cellular longevity, 2019. doi.org/10.1155/2019/4739450 
Zhang, Y., Lu, J., Ben, L. J., Zheng, L.X., \& Xu, P. F. (2019b). Optimization of cellulase-assisted extraction of total flavonoids from corn bract and evaluation of antioxidant and antibacterial activities. American Journal of Biochemistry and Biotechnology, 15(2), 61-74. doi.org/10.3844/ajbbsp.2019.61.74

Zhang, Y., Liu, Y., Yang, X., Cui, H., Xu, X., Mao, L., \& Zhou, H. (2019c). Antioxidant and immunomodulatory activities of Oviductus ranae in mice. Brazilian Journal of Pharmaceutical Sciences, 54. doi.org/10.1590/s2175-97902018000417751

Zheng, Y., Li, Y., \& Li, G. (2019). ACE-inhibitory and antioxidant peptides from coconut cake albumin hydrolysates: Purification, identification and synthesis. RSC advances, 9(11), 5925-5936.

doi.org/10.1039/C8RA10269D
Zhao, Y. J. (2018). Separation and characterization of antioxidant peptides from bass muscle and their protective effects against oxidative damage in HepG2 cells. South China University of Technology. doi.org/10.13982/j.mfst.1673-9078.2018.6.023

Zhang, Y., Zhu, K., Cui, H., Liu, Y., Lu, Y. F., Pan, H. W., .. \& Zhou, H. L. (2017). Toxicological evaluation of Oviductus ranae: Acute, sub-acute and genotoxicity studies in mice and rats. Journal of Ethno Pharmacology, 203, 101-109. doi.org/10.1016/j.jep.2017.03.032 